\title{
Lithium evolution in intermediate age and old open clusters: NGC 752 revisited ${ }^{\star}$
}

\author{
P. Sestito ${ }^{1}$, S. Randich ${ }^{2}$, and R. Pallavicini ${ }^{3}$ \\ 1 Dipartimento di Astronomia, Università di Firenze, Largo E. Fermi 5, 50125 Firenze, Italy \\ e-mail: sestito@arcetri.astro.it \\ 2 INAF - Osservatorio Astrofisico di Arcetri, Largo E. Fermi 5, 50125 Firenze, Italy \\ 3 INAF - Osservatorio Astronomico di Palermo, Piazza del Parlamento 1, 90134 Palermo, Italy
}

Received 6 May 2004 / Accepted 29 June 2004

\begin{abstract}
We present new high resolution spectroscopic observations of the intermediate age ( 2 Gyr) open cluster NGC 752. We investigate the Li vs. $T_{\text {eff }}$ distribution and we obtain a new accurate determination of the cluster metallicity. We compare the results for NGC 752 with other intermediate age and old clusters spanning the age range from the Hyades ( 0.6 Gyr) to NGC $188(\sim 6-8 \mathrm{Gyr})$. We find that NGC 752 has a solar iron content $([\mathrm{Fe} / \mathrm{H}]=+0.01 \pm 0.04)$, at variance with early reports of sub-solar metallicity. We find that NGC 752 is only slightly more Li depleted than the younger Hyades and has a Li pattern almost identical to that observed in the $\sim 2$ Gyr old IC 4651 and NGC 3680. As for the latter clusters, we find that NGC 752 is characterized by a tight $\mathrm{Li}$ vs. $T_{\text {eff }}$ distribution for solar-type stars, with no evidence for a Li spread as large as the one observed in the solar age solar metallicity M 67. We discuss these results in the framework of mixing mechanisms and Li depletion on the main sequence (MS). We conclude that the development of a large scatter in Li abundances in old open clusters might be an exception rather than the rule (additional observations of old clusters are required), and that metallicity variations of the order of $\sim \pm 0.2$ dex do not affect Li depletion after the age of the Hyades.
\end{abstract}

Key words. stars: abundances - stars: evolution - Galaxy: open clusters and associations: individual: NGC 752

\section{Introduction}

Lithium is present only in the outermost layers of a star, since its relatively low burning temperature $\left(T_{\mathrm{Li}}=2.5 \times 10^{6} \mathrm{~K}\right)$ allows Li destruction in stellar interiors. The amount of Li depletion strictly depends on the mixing mechanisms which transport material located at the bottom of the convective zone $(\mathrm{CZ})$ towards deeper layers, making $\mathrm{Li}$ a good tracer of the physical processes occurring during the various evolutionary phases of a star.

The current scenario for Li evolution in field stars of the solar neighborhood and in open clusters is very complex (see, e.g., the review of Pasquini 2000, and references therein) and so far the mechanism(s) driving Li depletion on the MS has/have not been clearly identified.

Observations of open clusters show that, at variance with the predictions of standard models (including only convection as a mixing process), Li depletion in solar-type stars is effective during MS evolution (e.g., Thorburn et al. 1993). In addition, metallicity seems to have a negligible effect on $\mathrm{Li}$ depletion at least up to the age of the Hyades (see Jeffries 2000, and

^ Based on observations collected at Telescopio Nazionale Galileo, La Palma, Canary Islands. references therein). Most surprisingly, the open cluster M 67 (solar age and solar metallicity) is characterized by a large dispersion in $\mathrm{Li}$ abundances for stars with similar effective temperatures (Pasquini et al. 1997; Jones et al. 1999). On the contrary, solar-type stars in the $\sim 2$ Gyr clusters NGC 3680 and IC 4651 (Randich et al. 2000) and in the 6-8 Gyr old cluster NGC 188 (Randich et al. 2003, hereafter R03) show a tight $\mathrm{Li}-T_{\text {eff }}$ relationship with the average $\mathrm{Li}$ distribution following that of the Li rich stars (the "upper envelope") of M 67. The only cluster with age intermediate between those of the Hyades and M 67 for which some evidence has been reported of a possible Li dispersion among solar analogs is NGC 752 (Hobbs \& Pilachowski 1986, hereafter HP86). It is worth mentioning that a large Li spread is also observed among field stars: for example, the Sun has $\log n(\mathrm{Li})=1.1$, much lower than the abundance predicted by standard models. At the same time there are stars older than the Sun (e.g. $\beta$ Hyi) with a much higher Li content (e.g., Pasquini et al. 1994).

The discrepancies between standard theoretical predictions and observational results clearly suggest that convection is not the only mixing process driving $\mathrm{Li}$ depletion: for this reason, models including extra-mixing mechanisms have been developed (see Pinsonneault 1997, and references therein), 
Table 1. Photometry, exposure times, effective temperatures, Li equivalent widths and Li abundances for the target stars. Asterisks denote $E W \mathrm{~s}$ corrected for the contribution of a nearby iron line (see text).

\begin{tabular}{ccccccc}
\hline \hline $\begin{array}{c}\text { No. } \\
\mathrm{P}\end{array}$ & $V$ & $B-V_{0}$ & $\begin{array}{c}\text { Exposure time } \\
(\mathrm{s})\end{array}$ & $\begin{array}{c}T_{\text {eff }} \\
(\mathrm{K})\end{array}$ & $\begin{array}{c}E W(\mathrm{Li}) \\
(\mathrm{m} \AA)\end{array}$ & $\log n(\mathrm{Li})_{N \mathrm{LTE}}$ \\
\hline 475 & 12.847 & 0.589 & $2200+1800$ & 5932 & $59 \pm 10$ & $2.48 \pm 0.16$ \\
520 & 12.850 & 0.535 & 3600 & 6151 & $67 \pm 12$ & $2.70 \pm 0.16$ \\
552 & 12.921 & 0.546 & $3600+3000$ & 6106 & $61 \pm 7$ & $2.62 \pm 0.14$ \\
648 & 12.108 & 0.550 & $3600+1800$ & 6089 & $24 \pm 8^{*}$ & $2.20 \pm 0.17$ \\
699 & 13.001 & 0.592 & $2000+2000$ & 5920 & $48 \pm 10$ & $2.37 \pm 0.17$ \\
701 & 13.060 & 0.655 & 3600 & 5677 & $42 \pm 12^{*}$ & $2.12 \pm 0.20$ \\
786 & 13.170 & 0.695 & $3600+1200$ & 5531 & $20 \pm 7$ & $1.67 \pm 0.23$ \\
859 & 13.200 & 0.635 & $2700+2700$ & 5753 & $26 \pm 7$ & $1.97 \pm 0.19$ \\
864 & 12.885 & 0.543 & 3600 & 6118 & $75 \pm 9$ & $2.74 \pm 0.15$ \\
889 & 12.802 & 0.516 & 3600 & 6231 & $78 \pm 11$ & $2.84 \pm 0.15$ \\
921 & 12.644 & 0.518 & 3600 & 6223 & $54 \pm 9$ & $2.65 \pm 0.15$ \\
964 & 12.912 & 0.547 & $3600+3600$ & 6102 & $64 \pm 9$ & $2.64 \pm 0.16$ \\
983 & 13.110 & 0.575 & 2000 & 5986 & $45 \pm 7$ & $2.38 \pm 0.15$ \\
993 & 13.590 & 0.673 & $3600+1800$ & 5611 & $20 \pm 6$ & $1.73 \pm 0.21$ \\
1012 & 12.417 & 0.504 & 3000 & 6282 & $74 \pm 10$ & $2.85 \pm 0.15$ \\
1107 & 13.660 & 0.625 & 1800 & 5791 & $24 \pm 5$ & $1.96 \pm 0.17$ \\
1284 & 12.893 & 0.642 & 1800 & 5726 & $42 \pm 9$ & $2.16 \pm 0.17$ \\
1365 & 13.290 & 0.675 & $2700+2700$ & 5603 & $48 \pm 8$ & $2.11 \pm 0.17$ \\
\hline
\end{tabular}

but these non-standard models are still poorly constrained, since they fail in reproducing quantitatively the observed features of Li evolution.

In this context, we have carried out high resolution spectroscopic observations of NGC 752, an unusually close (distance $\sim 360 \mathrm{pc}$, Friel 1995; $m_{V}-M_{V}=8.25 \pm 0.10$, Daniel et al. 1994, hereafter D94) 2 Gyr old cluster (Friel 1995), with estimated sub-solar metallicity $([\mathrm{Fe} / \mathrm{H}]=-0.15$, D94 $)$; the reddening towards the cluster is $E(B-V)=0.035$ (D94). NGC 752 is one of the best studied intermediate age open clusters, but surprisingly the only published $\mathrm{Li}$ data for this cluster date back to some 20 years ago (HP86; Pilachowski \& Hobbs 1988). Most important, the sample of HP86 includes very few solartype stars (while in Pilachowski \& Hobbs 1988, only F-type stars are studied); in spite of the small sample, as mentioned, HP86 found some evidence for the presence of a Li spread for solar analogs, although this issue was not discussed in their paper. We observed a much larger number of solar-type stars in NGC 752 in order to proof or dis-proof the possible presence of the scatter; moreover, the same target can be used to investigate the dependence of Li evolution on the metallicity by comparing with other $\sim 2$ Gyr old clusters: IC 4651 and NGC 3680, the first with over-solar iron content $([\mathrm{Fe} / \mathrm{H}] \sim+0.10 / 0.11$, Pasquini et al. 2004; Carretta et al. 2004), the second with $[\mathrm{Fe} / \mathrm{H}]=-0.17$ (Pasquini et al. 2001).

In this paper we present our new observations of solar-type stars in NGC 752, extending the previous survey of HP86. In Sect. 2 the observations and the abundance analysis are described; in Sects. 3 and 4 we present our results and a discussion, and finally in Sect. 5 we report our conclusions.

\section{Observations and abundance analysis}

\subsection{The sample}

Our sample includes 18 G-type stars selected from the $U B V$ photometry of D94; we selected only stars classified as members by D94, whose results are based on previous propermotion and radial velocities studies and on new radial velocities determined by them (see the discussion in the quoted reference). $B-V$ values range from 0.54 to 0.73 (approximately $\mathrm{F} 8$ to G8) and the visual magnitudes of the observed objects are in the range $V \sim 12.1-13.6$. Target stars and photometry are listed in Cols. 1-2, and 3 of Table 1; we used the identification numbers of Platais (1991) and we adopted the reddening quoted by $\mathrm{D} 94, E(B-V)=0.035$.

The observations were carried out during November 2002 and October 2003 at the Italian National Telescope Galileo (TNG), La Palma (Canary Islands), equipped with the SARG spectrograph (Gratton et al. 2000). Target stars were observed using the Red Cross-Disperser (spectral range 4960-10110 $\AA$ ) and the $O G 570$ filter, together with the mosaic of two EEV CCDs $\left(2048 \times 4096\right.$; pixel size $\left.13.5 \times 13.5 \mu \mathrm{m}^{2}\right)$; we employed a $2 \times 2 \mathrm{CCD}$ binning and slit aperture of $0.8^{\prime \prime}$, resulting in a nominal resolving power $R \sim 57000$. The total exposure times, listed in Col. 4 of Table 1 range from 1800 to $7200 \mathrm{~s}$, providing signal-to-noise $(S / N)$ ratios of $\sim 30-80$. 


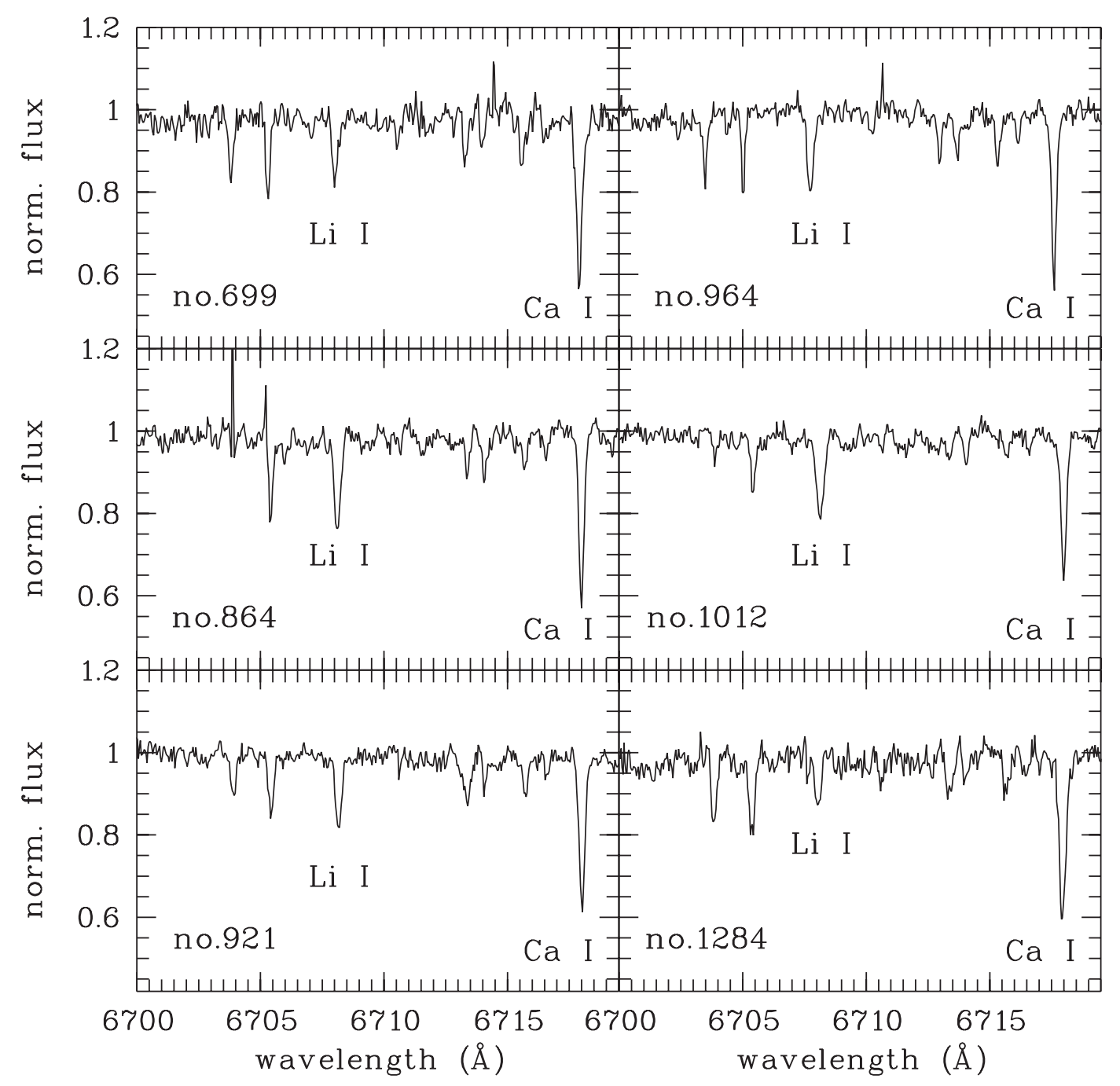

Fig. 1. Sample spectra in the Li region.

Data reduction was performed using the ECHELLE context within the package MIDAS and following the usual steps: first, we separated and flipped the two CCDs, then we performed bias subtraction, flat fielding, order definition, order extraction, sky and scattered light subtraction, and wavelength calibration. Figure 1 shows examples of NGC 752 spectra in the Li I $\lambda 6707.8 \AA$ region.

\subsection{Lithium}

Li abundance analysis was carried out as in R03; effective temperatures (listed in Col. 5 of Table 1) were estimated from dereddened $B-V$ colors using the calibration of Soderblom et al. (1993, hereafter S93): $T_{\text {eff }}=1808(B-V)_{0}^{2}-6103(B-$ $V)_{0}+8899 \mathrm{~K}$. The photometry of D94 is based on photoelectric data derived from six different sources combined and transformed into a common $B V$ system: typical errors in $B-V$ are $\sim 0.010-0.020 \mathrm{dex}$, with some stars having $\Delta(B-V)$ as large as 0.040 ; for several stars only one source is available and the error in $B-V$ is not quoted. In order not to underestimate the uncertainties in effective temperature and $\mathrm{Li}$ abundances, we adopted the conservative value $\Delta(B-V)= \pm 0.040$; this uncertainty in $B-V$ results into an error in $T_{\text {eff }}$ of $\pm 150 \mathrm{~K}$.

$\mathrm{Li}$ abundances $\left(\log n(\mathrm{Li})=12+\log N_{\mathrm{Li}} / N_{\mathrm{H}}\right)$ were determined from measured equivalent widths $(E W$, Col. 6$)$ of the Li I $\lambda 6707.8 \AA$ feature and effective temperatures by interpolation of the curves of growth (COG) of S93. Given the nominal resolving power and the low rotational velocities of the stars, the Li line should not be blended with the nearby $\mathrm{Fe}$ I feature at $\lambda 6707.44 \AA$; however, in the case of stars P648 and P701 (marked with asterisks in Table 1), due to low $S / N$ ratios we were not able to separate the $\mathrm{Fe}$ I feature from the $\mathrm{Li}$ I line, thus their $E W \mathrm{~s}$ were corrected for the Fe contribution using the prescriptions of S93: namely, $E W(\mathrm{Fe})=20(B-V)_{0}-3 \mathrm{~m} \AA$. We then applied non-local thermodynamic effect (NLTE) corrections to LTE Li abundances using the code of Carlsson et al. (1994). $\log n(\mathrm{Li})_{N L T E}$ are listed in Col. 7 of Table 1; uncertainties in $\mathrm{Li}$ abundances were derived by quadratically adding errors due to uncertainties in $E W \mathrm{~s}$ and $T_{\text {eff }}$.

In the following, we will compare our results for NGC 752 with those of HP86 and with the Li distribution of other clusters of different ages and/or metallicities (Hyades, Thorburn et al. 1993; NGC 3680 and IC 4651, Randich et al. 2000; M 67, 
Table 2. Photometry, effective temperatures, Li equivalent widths and $\mathrm{Li}$ abundances for the sample of HP86. Stars marked with asterisks are in common with us.

\begin{tabular}{ccccccc}
\hline \hline $\begin{array}{c}\text { No. } \\
\mathrm{P}\end{array}$ & $\begin{array}{c}\text { No. } \\
\mathrm{H}\end{array}$ & $V$ & $B-V_{0}$ & $\begin{array}{c}T_{\text {eff }} \\
(\mathrm{K})\end{array}$ & $\begin{array}{c}E W(\mathrm{Li}) \\
(\mathrm{m} \AA)\end{array}$ & $\log n(\mathrm{Li})_{N \mathrm{LTE}}$ \\
\hline 575 & 94 & 13.840 & 0.725 & 5425 & $<5$ & $<0.94$ \\
$701^{*}$ & 146 & 13.060 & 0.655 & 5677 & 45 & $2.15 \pm 0.20$ \\
790 & 185 & 12.267 & 0.494 & 6325 & 42 & $2.60 \pm 0.19$ \\
$859^{*}$ & 207 & 13.200 & 0.635 & 5753 & 7 & $1.38 \pm 0.20$ \\
917 & 227 & 14.100 & 0.895 & 4885 & $<12$ & $<0.82$ \\
$921^{*}$ & 229 & 12.644 & 0.518 & 6223 & 63 & $2.72 \pm 0.19$ \\
953 & 237 & 12.352 & 0.565 & 6027 & 43 & $2.39 \pm 0.21$ \\
1017 & 265 & 13.260 & 0.625 & 5791 & 15 & $1.75 \pm 0.20$ \\
\hline
\end{tabular}

Jones et al. 1999). In order to put all the data on a homogeneous scale, we reanalyzed the published equivalent widths with the method described above.

Stars selected from the HP86 sample are listed in Table 2, using the identification numbers of Platais (1991, Col. 1) and Heinemann (1926, Col. 2; the latter one adopted by HP86); there are three objects in common with our sample, marked with asterisks in Table 2. Effective temperatures (Col. 5) were recomputed by us starting from the photometry of D94 (Cols. 3 and 4). Note that we have selected only HP86 stars listed as members by D94 and with $T_{\text {eff }}$ cooler than $6500 \mathrm{~K}$, since stars warmer than $\sim 6300-6400 \mathrm{~K}$ should belong to the Li dip, which will not be discussed in this paper (see HP86; Pilachowski \& Hobbs 1988; Balachandran 1995). Column 6 shows the EWs of the $6708 \AA$ feature: HP86 did not take into account the possible presence of the $\mathrm{Fe}$ I contribution, thus we used their published $E W \mathrm{~s}$ without performing any correction. The nominal resolving power of HP86 spectra is $0.2 \AA$ and the correction should not be strictly necessary; however, at this resolution there could be a partial blending between the $\mathrm{Li}$ and Fe lines, thus NLTE Li abundances listed in Col. 7 of Table 2 might be slightly higher than the true abundances, especially for the coolest stars in the sample that are mostly affected by the Fe contribution (see the discussion in HP86). HP86 did not publish errors in $E W \mathrm{~s}$, but they quoted a $40 \%$ uncertainty in $\mathrm{Li}$ abundance $\left(N_{\mathrm{Li}} / N_{\mathrm{H}}\right)$ without considering the error due to uncertainties in $T_{\text {eff }}$. Errors listed in Col. 7 were computed by quadratically adding the two contributions.

\subsection{Metallicity}

The iron abundance of the cluster was derived using stars with high quality spectra, listed in Tables 3 and 4 . The analysis was carried out using MOOG (Sneden 1973, version December 2000) and Kurucz (1995) model atmospheres: as a first step, we adjusted $\log g f$ values (oscillator strength) by performing an inverse abundance analysis of the Sun. The solar spectrum was obtained at TNG during the second observing run (October 2003), using the same set-up as for our sample
Table 3. Stellar parameters for NGC 752 stars.

\begin{tabular}{cccccccc}
\hline \hline & P475 & P520 & P552 & P699 & P864 & P964 & P983 \\
\hline$T_{\text {eff phot }}$ & 5932 & 6151 & 6106 & 5920 & 6118 & 6102 & 5986 \\
$\xi_{\text {phot }}$ & 1.11 & 1.13 & 1.17 & 1.11 & 1.17 & 1.17 & 1.13 \\
$T_{\text {eff spec }}$ & 5982 & 6120 & 6130 & 5970 & 6060 & 6102 & 5930 \\
$\xi_{\text {spec }}$ & 1.20 & 1.30 & 1.30 & 1.25 & 1.18 & 1.17 & 0.92 \\
\hline
\end{tabular}

stars and pointing at the Moon; we assumed $\log n(\mathrm{Fe})_{\odot}=7.52$, $T_{\text {eff } \odot}=5770 \mathrm{~K}, \log g_{\odot}=4.44$ and $\xi_{\odot}=1.1 \mathrm{~km} \mathrm{~s}^{-1}$.

In our analysis of NGC 752 we assumed initial effective temperatures derived as described in Sect. 2.2. For all our sample stars we adopted the same surface gravity $\log g=$ 4.5 , while initial microturbulence values were derived as $\xi=$ $3.2 \times 10^{-4}\left(T_{\text {eff }}-6390\right)-1.3(\log g-4.16)+1.7($ Nissen 1981 ; Boesgaard \& Friel 1990). This relationship was obtained using a different $T_{\text {eff }}$ scale and different model atmospheres from those used in this paper (see Sect. 2.2); however, we note that (i) $\xi$ obtained from the above relationship are only assumed as initial values for the microturbulence; and (ii) as we will show below, there is a good agreement between the initial values and those derived by a "proper" spectroscopic analysis. Conservative random uncertainties for these two parameters are $\Delta \log g= \pm 0.3$ dex and $\Delta \xi= \pm 0.3 \mathrm{~km} \mathrm{~s}^{-1}$.

For each star we measured the $E W \mathrm{~s}$ of several Fe I lines in the wavelength range [5900, 6800] $\AA$ and we computed Fe abundances (see Table 4). When a trend of $\log n(\mathrm{Fe})$ vs. $E W$ or $E P$ (excitation potential) was found, we adjusted the photometric effective temperature and the initial value of microturbulence until the trend had disappeared. $T_{\text {eff }}$ and $\xi$ derived from the photometry ("phot") and from the Fe analysis ("spec") are listed in Table 3: note that the differences between the two sets of parameters are within the assumed errors, i.e. $\Delta T_{\text {eff }}= \pm 150 \mathrm{~K}$ (see Sect. 2.2) and $\Delta \xi= \pm 0.3 \mathrm{~km} \mathrm{~s}^{-1}$. In particular, all the differences between photometric and spectroscopic $T_{\text {eff }}$ listed in Table 3 are smaller than $60 \mathrm{~K}$, thus in our spectroscopic analysis we can assume a typical conservative error $\Delta T_{\text {eff }}= \pm 100 \mathrm{~K}$.

We did not correct surface gravities, since Fe I features are only slightly affected by this parameter; on the other hand, the dependence on $\log g$ is stronger in the case of Fe II lines, which however are very weak in our stars and cannot be used for an accurate analysis. In any case, for our sample stars which are all MS dwarfs, we do not expect surface gravities significantly different from the assumed value $\log g=4.5 \pm 0.3$.

In the last three rows of Table 4 the average $\log n(\mathrm{Fe})$ obtained for each star and the random errors $\sigma_{1}$ and $\sigma_{2}$ are listed: $\sigma_{1}$ is the standard deviation of the mean iron abundance, representative of errors mainly due to uncertainties in $E W \mathrm{~s}$ and $\log g f$, while $\sigma_{2}$ is the random error associated to uncertainties in $T_{\text {eff }}, \log g$ and $\xi$. We computed $\sigma_{2}$ by varying each parameter at a time and by quadratically adding the three related errors: we note that for all the stars $\sigma_{2}$ is much larger than $\sigma_{1}$, suggesting that the total random error is dominated by uncertainties in stellar parameters. 
Table 4. Fe abundances for stars in NGC 752 and for three stars in the Hyades. The average metallicities and their random errors are also given.

\begin{tabular}{|c|c|c|c|c|c|c|c|c|c|c|}
\hline \multirow{2}{*}{$\begin{array}{c}\text { Fe I line } \\
(\AA)\end{array}$} & \multicolumn{7}{|c|}{$\log n(\mathrm{Fe})$} & \multirow[b]{2}{*}{ vB21 } & \multirow[b]{2}{*}{ vB182 } & \multirow[b]{2}{*}{ vB187 } \\
\hline & P475 & P520 & P552 & P699 & P864 & P964 & P983 & & & \\
\hline 5930.18 & 7.54 & 7.55 & - & - & 7.57 & 7.53 & 7.59 & - & 7.60 & 7.63 \\
\hline 5934.65 & 7.60 & 7.55 & 7.62 & - & - & 7.57 & 7.52 & - & - & - \\
\hline 5956.69 & 7.60 & 7.54 & 7.63 & 7.47 & - & - & - & - & - & - \\
\hline 5976.77 & 7.48 & 7.48 & 7.48 & 7.61 & - & 7.55 & - & - & - & - \\
\hline 5984.81 & 7.55 & 7.44 & 7.45 & 7.57 & 7.44 & 7.55 & - & - & 7.66 & 7.72 \\
\hline 5987.07 & 7.63 & - & 7.59 & 7.64 & - & - & - & 7.72 & 7.70 & - \\
\hline 6024.06 & 7.44 & 7.55 & 7.58 & 7.40 & 7.52 & - & 7.51 & - & - & - \\
\hline 6056.00 & 7.45 & 7.50 & 7.62 & 7.57 & 7.46 & 7.53 & 7.61 & - & - & - \\
\hline 6078.49 & 7.60 & - & 7.62 & 7.49 & - & 7.53 & 7.65 & 7.71 & 7.70 & 7.70 \\
\hline 6136.99 & 7.46 & 7.53 & 7.51 & - & 7.46 & 7.50 & 7.56 & - & - & - \\
\hline 6157.73 & 7.57 & - & 7.63 & 7.48 & 7.47 & 7.50 & 7.43 & 7.63 & 7.68 & 7.65 \\
\hline 6187.99 & 7.54 & 7.51 & 7.57 & 7.51 & 7.49 & 7.57 & 7.58 & 7.72 & 7.70 & 7.68 \\
\hline 6200.31 & 7.55 & 7.52 & 7.47 & 7.44 & 7.58 & 7.50 & 7.63 & 7.68 & 7.66 & 7.69 \\
\hline 6315.81 & 7.55 & 7.54 & 7.50 & 7.56 & 7.48 & 7.49 & - & 7.62 & - & 7.69 \\
\hline 6322.69 & 7.58 & 7.52 & 7.50 & 7.60 & 7.55 & 7.58 & 7.62 & - & 7.60 & 7.72 \\
\hline 6330.85 & 7.52 & - & - & 7.42 & 7.52 & - & - & 7.62 & - & 7.61 \\
\hline 6336.82 & 7.57 & - & - & - & 7.50 & 7.51 & 7.49 & - & - & - \\
\hline 6344.15 & 7.46 & - & - & 7.49 & 7.53 & - & - & - & - & - \\
\hline 6469.19 & - & 7.55 & 7.63 & 7.56 & - & 7.52 & - & - & 7.70 & - \\
\hline 6498.94 & 7.49 & - & 7.52 & 7.61 & 7.53 & 7.51 & 7.55 & 7.60 & 7.61 & - \\
\hline 6574.23 & 7.58 & - & 7.59 & - & 7.45 & 7.50 & - & 7.60 & 7.61 & - \\
\hline 6609.11 & 7.46 & 7.44 & 7.49 & 7.57 & 7.48 & - & - & 7.69 & 7.70 & 7.71 \\
\hline 6627.55 & 7.56 & - & - & 7.58 & 7.43 & - & 7.59 & 7.63 & - & 7.62 \\
\hline 6703.57 & - & - & 7.59 & - & - & 7.65 & 7.58 & 7.63 & 7.67 & 7.64 \\
\hline 6725.36 & - & 7.49 & - & - & 7.59 & - & - & 7.70 & 7.65 & 7.66 \\
\hline 6726.66 & - & 7.55 & 7.59 & 7.57 & 7.46 & 7.60 & 7.59 & 7.70 & 7.66 & 7.68 \\
\hline 6750.15 & 7.47 & 7.45 & 7.61 & 7.39 & 7.49 & 7.57 & 7.53 & 7.58 & - & 7.62 \\
\hline average & 7.53 & 7.51 & 7.56 & 7.53 & 7.50 & 7.54 & 7.56 & 7.66 & 7.66 & 7.67 \\
\hline$\sigma_{1}$ & \pm 0.06 & \pm 0.04 & \pm 0.06 & \pm 0.07 & \pm 0.05 & \pm 0.04 & \pm 0.06 & \pm 0.05 & \pm 0.04 & \pm 0.04 \\
\hline$\sigma_{2}$ & \pm 0.10 & \pm 0.10 & \pm 0.10 & \pm 0.10 & \pm 0.10 & \pm 0.10 & \pm 0.10 & \pm 0.09 & \pm 0.09 & \pm 0.10 \\
\hline
\end{tabular}

We checked for the presence of systematic errors in the determination of $\log n(\mathrm{Fe})$ by performing the same analysis for three stars of the Hyades: this cluster has a well studied metallicity $([\mathrm{Fe} / \mathrm{H}]=+0.13$, e.g., Boesgaard \& Budge 1989) and can be safely used to put our results for NGC 752 on a self-consistent scale. Hyades stars (vB21, vB182 and vB187, listed in the last three Cols. of Table 4) were observed by us with UVES at VLT2 (the description of the data will be reported elsewhere). These spectra have $S / N \sim 200$ and a resolving power $R \sim 40000$, somewhat lower than SARG spectra: however, in our analysis we only used Fe features not blended at the UVES resolving power. Stellar parameters for the Hyades objects were derived consistently with our analysis of NGC 752: we found $T_{\text {eff }}=5142 \mathrm{~K}, \xi=0.86 \mathrm{~km} \mathrm{~s}^{-1}$ for $\mathrm{vB} 21, T_{\mathrm{eff}}=5079 \mathrm{~K}, \xi=0.84 \mathrm{~km} \mathrm{~s}^{-1}$ for $\mathrm{vB} 182$ and $T_{\text {eff }}=5339 \mathrm{~K}, \xi=0.92 \mathrm{~km} \mathrm{~s}^{-1}$ for $\mathrm{vB} 187$; for the three stars we assumed a surface gravity $\log g=4.5$.
Finally, we computed the weighted mean iron abundance using all the stars listed in Table 4. We found: a) $\log n(\mathrm{Fe})=$ $7.53 \pm 0.04$ or $[\mathrm{Fe} / \mathrm{H}]=+0.01 \pm 0.04$ for NGC 752 , i.e. the cluster has a nearly solar metallicity; b) $\log n(\mathrm{Fe})=7.66 \pm$ 0.06 , or $[\mathrm{Fe} / \mathrm{H}]=+0.14 \pm 0.06$ for the three Hyades stars, very similar to the value published by Boesgaard \& Budge (1989). In the computation of the weighted mean, we assumed $\sigma=\sqrt{\sigma_{1}^{2}+\sigma_{2}^{2}}$ for each star. We note that the $1 \sigma$-errors on the mean abundances are much lower than the typical $\sigma$ for each star, again suggesting that our adopted uncertainties are conservative. Systematic errors such as the model atmospheres and the line list used, together with possible biases due to different methods of $T_{\text {eff }}$ estimation and cluster reddening uncertainties, are in principle by far more important. However, the $[\mathrm{Fe} / \mathrm{H}]$ obtained for the Hyades, which is in very good agreement with previous estimates, allows us to exclude the presence of large systematic errors. 


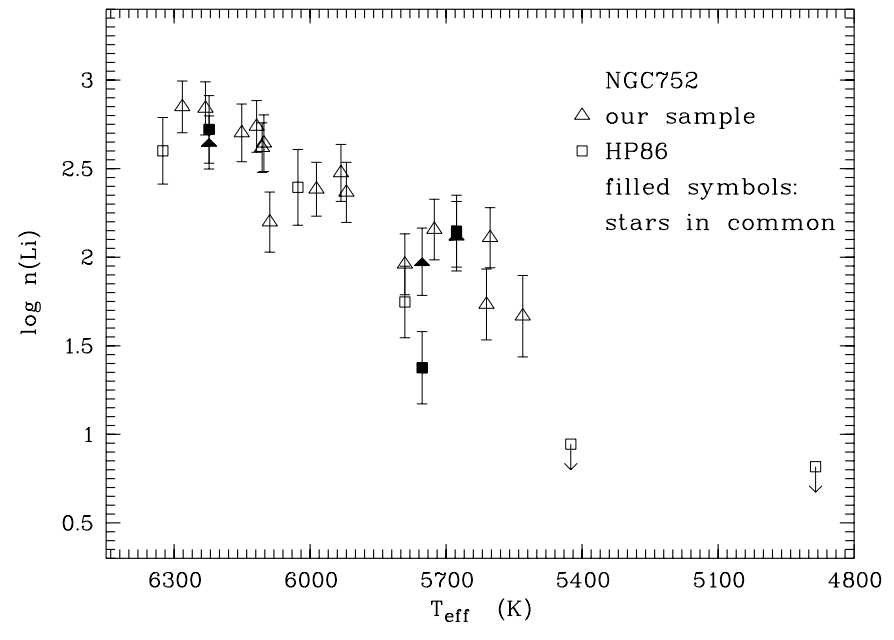

Fig. 2. $\log n(\mathrm{Li})$ vs. $T_{\text {eff }}$ for NGC 752 - comparison between our sample (triangles) and that of HP86 (squares). Filled symbols represent stars in common between the two samples; down-pointing arrows indicate upper limits in $\log n(\mathrm{Li})$.

In Sect. 1 we pointed out that D94 assumed a sub-solar metallicity for the cluster $([\mathrm{Fe} / \mathrm{H}]=-0.15 \pm 0.05)$, choosing this value from a "by eye" average of various published metallicities. The most recent investigations are the spectroscopic studies of Hobbs \& Thorburn (1992) and Friel \& Janes (1993). The first authors found $[\mathrm{Fe} / \mathrm{H}]=-0.09 \pm 0.05$ from 8 solartype stars in the sample of HP86; Friel \& Janes (1993) found $[\mathrm{Fe} / \mathrm{H}]=-0.16 \pm 0.05$, based on a sample of nine low resolution spectra $(\Delta \lambda=4 \AA)$ of NGC 752 giant stars. The result of Hobbs \& Thorburn (1992) is more reliable, since it is based on high resolution spectra $(\Delta \lambda=0.2 \AA)$, but in any case their average $[\mathrm{Fe} / \mathrm{H}]$ is rather different from our estimate.

On the other hand, Chaboyer et al. (1995) adopted a solar iron content for the cluster in a comparison between the HP86 sample and theoretical models including rotation and diffusion. Our estimate of the metallicity of NGC 752 is perfectly self-consistent and based on the comparison with the Hyades, for which we found $[\mathrm{Fe} / \mathrm{H}]$ in agreement with Boesgaard \& Budge (1989). Therefore, we can safely assume a nearly solar metallicity for the cluster.

\section{Results}

Figure 2 shows the $\mathrm{Li}$ distribution $\left(\log n(\mathrm{Li})\right.$ vs. $\left.T_{\text {eff }}\right)$ of NGC 752: our sample is compared to that of HP86. Li abundances for the three stars in common between the two samples are plotted as filled symbols: there is a very good agreement for P701 and P921 ( $T_{\text {eff }}=5677 \mathrm{~K}$ and $T_{\text {eff }}=6223 \mathrm{~K}$, respectively), while in the case of P859 $\left(T_{\text {eff }}=5753 \mathrm{~K}\right)$ there is a large discrepancy between the two measurements. HP86 reported $E W=7 \mathrm{~m} \AA$ for this object, while we measure $26 \pm$ $7 \mathrm{~mA}$; as mentioned in Sect. 2.2, HP86 did not publish the values of the errors in $E W \mathrm{~s}$, but they indicated a mean uncertainty of about $40 \%$ in $N_{\mathrm{Li}} / N_{\mathrm{H}}$ (that we interpreted to be entirely due to the uncertainty in $E W$ ). For star P859, this would translate into an error of $\sim 0.15 \mathrm{dex}$ in $\log n(\mathrm{Li})$, or $\sim 3 \mathrm{~m} \AA$ in $E W$, thus it is evident that the two measurements are in complete disagreement. This discrepancy could be due to the lower resolution and possibly to a less than optimum $S / N$ ratio of HP86 spectra: for this reason, in the following we will use our measurements for the stars in common.

The hottest NGC 752 stars $\left(T_{\text {eff }}>6000 \mathrm{~K}\right)$ are Li rich, since they have shallow convective envelopes that do not allow a fast $\mathrm{Li}$ destruction: their $\log n(\mathrm{Li})$ values are only $\sim 2-3$ times lower than the meteoritic abundance $\left(\log n(\mathrm{Li})_{0}=3.1-3.3\right.$, e.g. Grevesse \& Sauvel 1998), which is thought to be representative of the initial $\mathrm{Li}$ content. $\mathrm{Li}$ abundances decrease towards lower effective temperatures and, as expected, the coolest objects in the sample $\left(T_{\text {eff }}<5500 \mathrm{~K}\right)$ have only upper limits in $\log n(\mathrm{Li})$ since they are characterized by deep CZs which allow a fast $\mathrm{Li}$ depletion to occur. Solar analogs $\left(5600 \lesssim T_{\text {eff }} \lesssim 6000\right)$ cluster around a "plateau": more in detail, stars with $T_{\text {eff }} \sim 6000 \mathrm{~K}$ have average Li abundances of $\sim 2.4$, while stars around $\sim 5700 \mathrm{~K}$ have $\log n(\mathrm{Li}) \sim 2$. The cluster does not appear to be characterized by a large amount of spread, as discussed below.

In Fig. 3 NGC 752 is compared to various clusters. In panel a) we report a comparison between the NGC 752 merged sample (our stars+HP86, filled triangles) and the $600 \mathrm{Myr}$ old Hyades (Thorburn et al. 1993, starred symbols). G-type stars in NGC 752 are somewhat more Li depleted than their Hyades counterparts, but not as much as expected given the difference in age: $\log n(\mathrm{Li})$ values in NGC 752 are on average less than a factor 2 lower than those observed in the Hyades, while the difference between $\mathrm{Li}$ abundances in the Hyades and those of young clusters (e.g. the Pleiades) is much larger. Assuming that $\mathrm{Li}$ is effectively destroyed during the MS phase, as shown by observations but at variance with standard model predictions, the behavior of NGC 752 suggests that Li depletion begins to slow down after the age of the Hyades (see R03), while for younger clusters the rate of Li burning with age for G-type stars is nearly linear (Sestito et al. 2003).

In Fig. 3b NGC 752 is compared to IC 4651 and NGC 3680 (Randich et al. 2000; open circles and asterisks): the three $\mathrm{Li}$ distributions are almost indistinguishable. We remind that IC 4651 and NGC 3680 have similar ages ( 2 Gyr) but different metallicities with respect to NGC 752; therefore, age seems to be the main parameter driving Li depletion during the first 2 Gyr of stellar evolution, while the iron content might only have second order effects (see Sect. 4.2).

Finally, we show a comparison between NGC 752 and the solar-age M 67 (Jones et al. 1999; Fig. 3c - open lozenges). First of all, we note that the average Li distribution of NGC 752 lies on the upper envelope of the latter cluster; second, the most important feature emerging from this plot is that NGC 752 is characterized by a rather tight $\mathrm{Li}$ vs. $T_{\text {eff }}$ distribution, more similar to that of the Hyades than to the pattern of M 67.

In the latter cluster about $40 \%$ of the stars have severely destroyed Li, having abundances 5-10 times lower than the remaining ones. On the contrary, in NGC 752 there are only a few objects slightly deviating from the average Li distribution and they should not be considered as representative of a large Li spread. Li abundances slightly lower than the general trend could be due to poor $S / N$ ratios or to possible errors in the photometry. Therefore, we stress that whereas M 67 is 

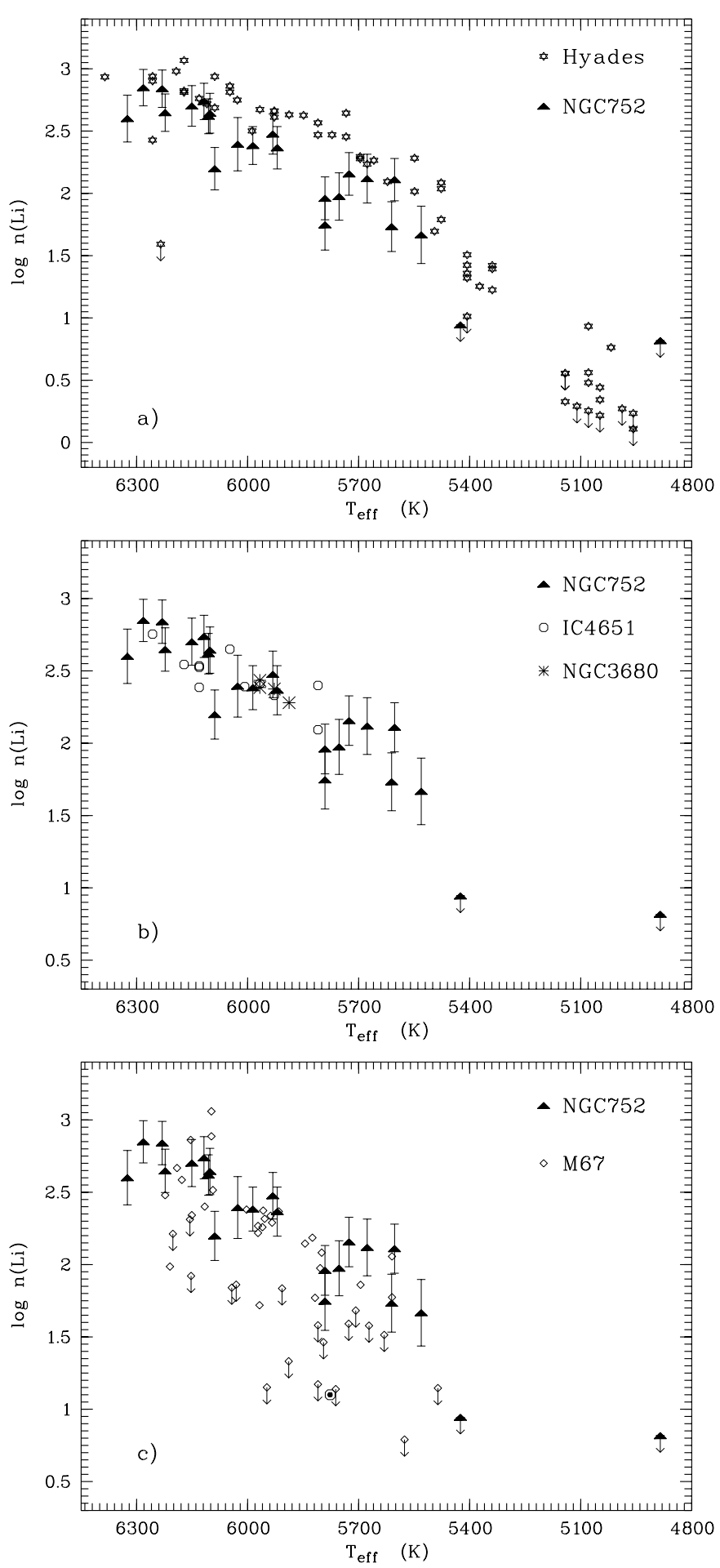

Fig. 3. $\log n(\mathrm{Li})$ vs. $T_{\text {eff }}-$ comparison between the NGC 752 merged sample (our stars+HP86, filled triangles) and other clusters: a) the Hyades (Thorburn et al. 1993); b) IC 4651 and NGC 3680 (Randich et al. 2000); c) M 67 (Jones et al. 1999).

clearly characterized by a large Li dispersion, having a considerable fraction of the stars that suffered a large Li destruction, NGC 752 has a quite "regular" Li distribution with all the stars having preserved a relatively high Li content.

\section{Discussion}

In Sect. 3 we pointed out two main results from our analysis of NGC 752: a) the Li vs. $T_{\text {eff }}$ distribution of the cluster is not characterized by a significant dispersion; b) Li depletion seems not to be metal dependent at the age of $\sim 2 \mathrm{Gyr}$.

\subsection{The star-to-star scatter in Li abundances}

The first goal of this study was to determine whether a starto-star scatter in Li abundances exists among solar-type stars of NGC 752. In summary, in the last decade the presence of a considerable Li spread has been ascertained among solar-like field stars (Pasquini et al. 1994) as well as in the solar age, solar metallicity open cluster M 67 (e.g., Pasquini et al. 1997; Jones et al. 1999). Randich et al. (2000) showed that no spread is present in the $\sim 2$ Gyr old clusters NGC 3680 and IC 4651 and suggested (among other possibilities) that the dispersion might develop after this age. The more recent investigation by R03 demonstrated however that also the 6-8 Gyr old NGC 188 does not show a spread in Li abundances. NGC 188 is one of the oldest known open clusters and the oldest open cluster in which $\mathrm{Li}$ has been studied; R03 showed that the Li distribution of this cluster is similar to that of NGC 3680 and IC 4651: solar-type stars indeed lie on the upper envelope of M 67, i.e. they are quite Li rich in spite of the old age of NGC 188. This feature might indicate a slowing down of $\mathrm{Li}$ depletion at ages larger than $\sim 2$ Gyr, already discussed in a detailed way in the quoted reference.

On the other hand, HP86 data for NGC 752 showed some indication for the presence of a Li dispersion among solar-type stars, but the sample was rather sparse. As shown by our analysis, based on new high resolution observations of NGC 752, the cluster is characterized by a tight $\mathrm{Li}-T_{\text {eff }}$ relationship with only a few stars slightly deviating from the mean trend, which cannot be considered as statistically representative of a scatter. We can demonstrate this by considering the temperature range $T_{\text {eff }} \sim$ [5500-6300] where M 67 shows the Li spread (cooler stars have to be excluded since they are exhausting their $\mathrm{Li}$ content, as expected due to the deep CZs): in this region we have 20 NGC 752 stars and at most 2 objects are slightly deviating from the average Li pattern (P648 and P1017), while in the case of M 67 we conservatively assume that $30 \%$ of stars are over-depleted. Thus, if a similar fraction should hold for both the clusters, using a binomial distribution we have a probability $\mathrm{P}(2 / 20)=2.78 \times 10^{-2}$ of finding two Li poor stars in our sample, which is between $2 \sigma$ and $3 \sigma$. If we had considered only 1 NGC 752 star as over-depleted and a more realistic fraction of $\mathrm{Li}$ poor stars in M 67 equal to $40 \%$, we would have found $\mathrm{P}(1 / 20)=4.87 \times 10^{-4}$ (about $4 \sigma$ ). In both cases we can exclude the presence of over-depleted stars in NGC 752; moreover, we stress that the deviation of stars P648 and P1017 from the NGC $752 \mathrm{Li}$ distribution is not quantitatively comparable with the spread observed in M 67: P648 and P1017 are indeed a factor $\sim 2$ more Li depleted than NGC 752 stars of similar temperatures, while stars in the lower envelope of M 67 suffered a depletion of factors $\sim 5-10$ with respect to their Li rich counterparts. 
In other words, our analysis points out that (i) M 67 is so far the only old open cluster showing a Li spread similar to that observed among nearby field stars, i.e. the development of a dispersion might be an exception rather than the rule in intermediate age/old open clusters; (ii) $\mathrm{Li}$ is not a good age tracer, at least for open clusters older than $\sim 1 \mathrm{Gyr}$, since Li depletion in G-type cluster stars appears to slow down after the age of the Hyades, but at the same time stars in the field and in M 67 exist for which Li destruction is unexpectedly very fast.

Several attempts have been made over the last years in order to understand the possible physical mechanisms responsible for the spread in M 67 (see the references quoted below for further details). For example Jones et al. (1999) proposed the presence of mixing driven by rotation (models of Pinsonneault et al. 1990; Chaboyer et al. 1995; Martín \& Claret 1996), while Pasquini et al. (1994) noticed that also the presence of mass loss might play a role in the appearing of the scatter (models of Swenson \& Faulkner 1992). García López et al. (1988) suggested that M 67 could contain two sub-clusters with different ages and/or chemical composition. A scatter in heavy element abundances might indeed affect the tachocline diffusion processes related to light element burning (Piau et al. 2003).

None of the proposed explanation sounds satisfactory, since they are all in contradiction with the following empirical evidences: observations of the Li-rotation distribution in young clusters (e.g. Pleiades, S93; Alpha Persei, e.g. Randich et al. 1998); the absence of a $\mathrm{Be}-\mathrm{Li}$ correlation (Randich et al. 2002) and the absence of a large scatter in heavy element abundances in M 67 (Randich et al. 2004, in preparation). Moreover, the models of Swenson \& Faulkner (1992) fail in quantitatively reproducing the amount of mass loss suffered by the Sun and stars in clusters as young as the Hyades.

We prefer to attribute the cause of the Li dispersion observed in M 67 to a possible inhomogeneity of the cluster, similarly to García López et al. (1988), although the origin of this inhomogeneity is not clear and, as mentioned, it seems not to be related to heavy element abundances. In any case M 67 appears to be a peculiar cluster, at least considering the - admittedly small - dataset for intermediate age/old open clusters. In parallel, the problem remains of understanding the similar scatter observed among solar-like field stars: in particular, the most puzzling issue is the very poor amount of Li present in the Sun which cannot be explained by any kind of model.

\subsection{Li depletion and iron content}

One of the unsolved discrepancies between observations of $\mathrm{Li}$ in open clusters and theoretical predictions regards the dependence of Li depletion on metallicity. In fact, as already mentioned, standard models predict an increase of the opacity and therefore of the depth of the CZ (meaning a faster Li destruction) as the metallicity increases. We mention that also the content of oxygen and $\alpha$ elements can affect the opacity (Piau \& Turck-Chiéze 2002).

On the other hand the empirical picture does not support this point of view, at least as far as zero-age MS stars in young clusters are concerned. For example, Jeffries \& James (1999) carried out a survey of Blanco $1(100 \mathrm{Myr},[\mathrm{Fe} / \mathrm{H}]=+0.14)$ and they compared it to the similar age Pleiades $([\mathrm{Fe} / \mathrm{H}] \sim$ solar $)$, showing that the two clusters have similar Li patterns, i.e. have suffered the same amount of pre-MS Li depletion in spite of the different iron contents.

Our analysis of $\mathrm{Li}$ and $\mathrm{Fe}$ abundances in the $2 \mathrm{Gyr}$ old NGC 752, which we found to have a solar metallicity, allows us to extend the previous results to older ages. Figure $3 b$ shows that G-type stars in NGC 752 have a Li pattern almost identical to that of stars with the same effective temperature in IC $4651([\mathrm{Fe} / \mathrm{H}]=+0.10)$ and NGC $3680([\mathrm{Fe} / \mathrm{H}]=$ $-0.17)$. The metallicities of the three clusters have been derived with different codes/methods, and for this reason the comparison could in principle be affected by systematic errors; however, we note that the three estimates are all on the same scale. In fact, Pasquini et al. (2004), which estimated $[\mathrm{Fe} / \mathrm{H}]=+0.10$ for IC 4651 with the code of Spite (1967), obtained the same result by carrying out the analysis with MOOG. Furthermore, they analyzed some Hyades stars which turned out to have $[\mathrm{Fe} / \mathrm{H}]$ in agreement with our estimate and with Boesgaard \& Budge (1989). Pasquini et al. (2001) found instead $[\mathrm{Fe} / \mathrm{H}]=+0.06$ for two Hyades stars, using the code of Carretta \& Gratton (1997), and for this reason they scaled by $\sim 0.1$ dex the metallicity obtained for NGC 3680 , quoting at the end a best value of $[\mathrm{Fe} / \mathrm{H}]=-0.17$.

In other words, if we assume that the iron contents of the three clusters were derived correctly and are on the same scale, as it seems to be, we can say that variations within $\sim \pm 0.15 / 0.20 \mathrm{dex}$ in $[\mathrm{Fe} / \mathrm{H}]$ do not affect $\mathrm{Li}$ depletion, even at ages older than that of the Hyades.

We can also exclude a possible dependence of the Li spread on the iron content as suggested by the Li patterns of NGC 752, M 67, and NGC 188 (see Fig. 3c, and R03): the three clusters represent an age sequence with fixed solar $[\mathrm{Fe} / \mathrm{H}]$, but only M 67 shows a dispersion.

\section{Summary and conclusions}

We have presented new high resolution spectroscopic observations of 18 G-type stars in the $\sim 2$ Gyr old open cluster NGC 752, obtained with SARG at TNG. Our data extend the previous investigation by Hobbs \& Pilachowski (1986) which was based on a smaller sample.

We investigated the $\mathrm{Li}$ vs. $T_{\text {eff }}$ distribution in this cluster, as well as the metallicity, and we obtained the following results:

a) NGC 752 turns out to have a nearly solar iron content $([\mathrm{Fe} / \mathrm{H}]=+0.01 \pm 0.04)$, at variance with other results in the literature, which reported a sub-solar metallicity.

b) NGC 752 is only slightly more Li depleted than the younger Hyades and has a $\mathrm{Li}$ pattern almost identical to that observed in the 2 Gyr clusters IC 4651 and NGC 3680.

c) As the other 2 Gyr clusters and as the older NGC 188, NGC 752 is characterized by a rather tight Li distribution for solar-type stars, i.e. these clusters do not show the large Li spread shown by the solar age M 67. Li abundances of NGC 752 stars are similar to those of the upper envelope (Li rich stars) of M 67. 
This evidence leads to the conclusions that:

(i) As far as the empirical picture is concerned, M 67 is so far the only cluster showing a large Li spread for solartype stars: as such, it appears to be unusual among intermediate age and old open clusters. This star-to-star scatter is similar to that observed among nearby field stars, which suggests that M 67 might represent an inhomogeneous sample.

(ii) Since IC 4651 and NGC 3680 have similar ages but different $[\mathrm{Fe} / \mathrm{H}]$ with respect to NGC 752 (see Sect. 4.2) we conclude that variations of the metallicity within $\sim \pm 0.2$ dex seems to not affect Li evolution after the age of the Hyades, extending the results found by other authors for younger clusters.

(iii) The comparison of the 2 Gyr clusters with the Hyades, with the upper envelope of M 67 and with NGC 188 allowed us to confirm that Li destruction in G-type stars appears to slow down for very old clusters. This result was already discussed by Randich et al. (2003) and our investigation added a new sample, providing a higher statistics.

Our conclusions are based on a small sample of old open clusters: three 2 Gyr clusters, the solar age M 67 and the very old NGC 188; in order to further investigate the above problems, i.e. the dispersion in $\mathrm{M} 67$, the Li-metallicity relationship, the slowing down of Li depletion, new observational data are clearly required which would provide a higher statistical basis. In this respect, we mention that we have started a project aimed at acquiring $\mathrm{Li}$ data for a large sample of open clusters older than 1 Gyr. On the other hand, progress in the theoretical models is also required in order to understand the more complete observational scenario that is coming out from the new observations.

Acknowledgements. P.S. and S.R. acknowledge support from the Italian Ministero dell'Istruzione, Università e Ricerca through a COFIN grant. P.S. is grateful to the TNG staff for the help during the observations. We thank the referee, Dr. R. D. Jeffries for his useful comments.

\section{References}

Balachandran, S. 1995, ApJ, 446, 203

Boesgaard, A. M., \& Budge, K. G. 1989, ApJ, 338, 875

Boesgaard, A. M., \& Friel, E. D. 1990, ApJ, 351, 467

Carlsson, M., Rutten, R. J., Bruls, J. H. M., \& Schukina, N. G. 1994, A\&A, 288, 860

Carretta, E., \& Gratton, R. G. 1997, A\&AS, 121, 95
Carretta, E., Bragaglia, A., Gratton, R. G., \& Tosi, M. 2004 [arXiv: astro-ph/0404298

Chaboyer, B., Demarque, P., \& Pinsonneault, M. H. 1995, ApJ, 441, 876

Daniel, S. A., Latham, D. W., Mathieu, R. D., \& Twarog, B. A. 1994, PASP, 106, 281

Friel, E. D. 1995, ARA\&A, 33, 381

Friel, E. D., \& Janes, K. A. 1993, A\&A, 267, 75

García López, R. J., Rebolo, R., \& Beckmann, J. E. 1988, PASP, 100, 1489

Gratton, R., Bonanno, G., Bruno, P., et al. 2000, Proc. SPIE, 4008, 244

Grevesse, N., \& Sauval, A. J. 1998, SSrv, 85, 161

Heinemann, R. 1926, Astr. Nach., 227, 213

Hobbs, L. M., \& Pilachowski, C. 1986, ApJ, 309, L17

Hobbs, L. M., \& Thorburn, J. A. 1992, AJ, 104, 669

Jeffries, R. D. 2000, in Stellar Clusters and Associations: Convection, Rotation, and Dynamos, ed. R. Pallavicini, G. Micela, \& S. Sciortino, ASP Conf. Ser., 198, 245

Jeffries, R. D., \& James, D. J. 1999, ApJ, 511, 218

Jones, B. F., Fischer, D., \& Soderblom, D. R. 1999, AJ, 117, 330

Kurucz, R. L. 1995, ApJ, 452, 102

Martín, E., \& Claret, A. 1996, A\&A, 306, 408

Nissen, P. 1981, A\&A, 97,145

Pasquini, L. 2000, in The Light Elements and their Evolution, ed. L. da Silva, M. Spite, \& J. R. de Medeiros, IAU Symp., 198, 269

Pasquini, L., Liu, Q., \& Pallavicini, R. 1994, A\&A, 287, 191

Pasquini, L., Randich, S., \& Pallavicini, R. 1997, A\&A, 325, 535

Pasquini, L., Randich, S., \& Pallavicini, R. 2001, A\&A, 374, 1017

Pasquini, L., Randich, S., Zoccali, M., et al. 2004, A\&A, 424, 951

Piau, L., \& Turck-Chiéze, S. 2002, ApJ, 566, 419

Piau, L., Randich, S., \& Palla, F. 2003, A\&A, 408, 1037

Pilachowski, C., \& Hobbs, L. M. 1988, ApJ, 334, 734

Pinsonneault, M. 1997, ARA\&A, 35, 557

Pinsonneault, M., Kawaler, S. D., \& Demarque, P. 1990, ApJS, 74, 501

Platais, I. 1991, A\&AS, 87, 69

Randich, S., Martín, E., García López, R. J., \& Pallavicini, R. 1998, A\&A, 333, 591

Randich, S., Pasquini, L., \& Pallavicini, R. 2000, A\&A, 356, L25

Randich, S., Primas, F., Pasquini, L., \& Pallavicini, R. 2002, A\&A, 387,222

Randich, S., Sestito, P., \& Pallavicini, R. 2003, A\&A, 399, 133

Randich, S., Primas, F., Sestito, P., Pasquini, L., \& Pallavicini, R. 2004, in preparation

Sestito, P., Randich, S., Mermilliod, J.-C., \& Pallavicini, R. 2003, A\&A, 407, 289

Sneden, C. A. 1973, ApJ, 184, 839

Soderblom, D. R., Jones, B. F., Balachandran, S., et al. 1993, AJ, 106, 1059

Spite, M. 1967, Ann. Astrophys., 30, 211

Swenson, F. J., \& Faulkner, J. 1992, ApJ, 395, 654

Thorburn, J. A., Hobbs, L. M., Deliyannis, C. P., \& Pinsonneault, M. H. 1993, ApJ, 415, 150 